\title{
Editorial Introduction
}

This issue of the Independent Journal of Management \& production (IJM\&P) features a selection of articles submitted and revised until October 2013. Observed that works are the fruit of research and publications of undergraduate, postgraduate and entrepreneurs.

The publication also counts on the work of researchers from various parts of the world, which have undergone a process of peer review.

As chief editor of $I J M \& P, I$ am indebted to all members of the editorial board and reviewers, which contributed to achieving a very decent job during the evaluation and revision. And that has contributed to the Journal in recognition of the international scientific community.

And with all the authors, who trusted the results of their research and publications to the scrutiny of editors and reviewers who are part of our Journal.

In the second half of 2013, we had good news about the journal and we are sharing some of them here. For example, we received the following impact factors year 2012:

- Universal Impact Factor $\{U I F\}$

0,4285

2012

- Research Bible 0,42 2012

- Global Impact Factor (GIF)

0,512

2012

Here is a list of reviewers who evaluated papers for the Journal between 2010 and 2013. 
INDEPENDENT JOURNAL OF MANAGEMENT \& PRODUCTION (IJM\&P)

http://www.ijmp.jor.br

v. 5, n. 1, October - January 2014.

ISSN: 2236-269X

DOI: 10.14807/ijmp.v5i1.157

\begin{tabular}{|c|c|c|}
\hline Title & Name & Country \\
\hline Msc. & Abel Anibal Del Río & $\mathrm{CO}$ \\
\hline Dr. & Adeyemi Oluseyi Ewetade & NG \\
\hline Prof. Ph.D. & Adriana Burlea Schiopoiu & RO \\
\hline Ph.D. Student & Aline Marian Callegaro & $\mathrm{BR}$ \\
\hline Msc. & Alvaro Luiz Neuenfeldt Júnior & $\mathrm{BR}$ \\
\hline Dr. & Amarildo Da Cruz Fernandes & BR \\
\hline Dr. & Amran Awang & MY \\
\hline Msc. & Ana Maria Magalhães Correia & $\mathrm{BR}$ \\
\hline Msc. & Anderson Catapan & $\mathrm{BR}$ \\
\hline Dr. & Andre Luis Korzenowski & $\mathrm{BR}$ \\
\hline Doctoral Candidate & Andre Vilares Morgado & PT \\
\hline $\mathrm{Mr}$ & Andreas Hagen & SE \\
\hline Dr. & Andreas Dittmar Weise & $\mathrm{BR}$ \\
\hline Doctoral Student & Andréia De Abreu & $\mathrm{BR}$ \\
\hline Dr. & Antonio Silva & $\mathrm{BR}$ \\
\hline Dr. & Bassam Edmond Badran & SY \\
\hline Prof. Msc. & Carlos Antonio Pizarro Louzada & $\mathrm{BR}$ \\
\hline Ph.D. & Carmen Martins Marques & PT \\
\hline Prof. Msc. & David Custódio De Sena & $\mathrm{BR}$ \\
\hline Msc. & Diego Pacheco & BR \\
\hline Msc. & Ernani Da Cunha Ferreira & $\mathrm{BR}$ \\
\hline Dn. & Euro Marques Júnior & BR \\
\hline Msc. & Everton Drohomeretski & $\mathrm{BR}$ \\
\hline Dr. & Fabrizio Baldassarre & IT \\
\hline Prof. Dr. & Fernando Celso Campos & $\mathrm{BR}$ \\
\hline Prof. LD. & Fernando Augusto Silva Marins & BR \\
\hline Dr. & Florian Ion Petrescu & RO \\
\hline Dr. & Francisco José Santos Milreu & BR \\
\hline Dn. & Gerson Rossi Dos Santos & BR \\
\hline Dna. & Geslaine Frimaio Silva & $\mathrm{BR}$ \\
\hline Ph.D. Candidate & Gordon Monday Bubou & NG \\
\hline MP. & Graciela Dias Coelho Jones & BR \\
\hline Prof. Ph.D. Wg. Cdr. & Gulshan Kumar Bajwa & IN \\
\hline Mr. & Gustavo Henrique Silva De Souza & BR \\
\hline Mr. & Harlan D. Whatley & $\mathrm{CN}$ \\
\hline Msc. & Hector Ricardo Formento & AR \\
\hline Prof. Dr. & Heitor Luiz Murat De Meirelles Quintella & $\mathrm{BR}$ \\
\hline Dr. & Hemant Bamoriya & IN \\
\hline Dr. & Henrique Martins Rocha & BR \\
\hline Profa. Dra. & leda Kanashiro Makiya & $\mathrm{BR}$ \\
\hline Ph.D. & Irene Samanta & GR \\
\hline MA. & Jamal Mohammed & $\mathrm{GH}$ \\
\hline
\end{tabular}


INDEPENDENT JOURNAL OF MANAGEMENT \& PRODUCTION (IJM\&P)

http://www.ijmp.jor.br

v. 5, n. 1, October - January 2014.

ISSN: 2236-269X

DOI: 10.14807/ijmp.v5i1.157

\begin{tabular}{|c|c|c|}
\hline Dr. & Jimmy Francisco Barangay & $\mathrm{PH}$ \\
\hline Dr. & João Carlos Colmenero & $\mathrm{BR}$ \\
\hline Ph.D. & Joao Correia Leitao & PT \\
\hline Ph.D. & Joel Kimeli Cherus & KE \\
\hline Prof. Dr. & Jose Paulo Fusco & $\mathrm{BR}$ \\
\hline Dr. & José Barrozo De Souza & $\mathrm{BR}$ \\
\hline Dr. & Jose Alberto Carvalho Dos Santos Claro & $\mathrm{BR}$ \\
\hline Doctoral Candidate & Joshin John & IN \\
\hline Dr. & Juanjo Boté & ES \\
\hline MBA. & Kaveh Hasani & IR \\
\hline Dr. & Kent R Acheson & US \\
\hline Dr. & Kirti Dutta & IN \\
\hline Dr. & Lavanchawee Sujarittanonta & $\mathrm{TH}$ \\
\hline Ph.D. & Liviu Drugus & RO \\
\hline Prof. Dr. & Luiz Flavio Autran Monteiro Gomes & $\mathrm{BR}$ \\
\hline Dr. & Manuj Darbari & IN \\
\hline Mn. & Mara Regina Dos Santos Barcelos & $\mathrm{BR}$ \\
\hline Dr. & Marcelo Battesini & $\mathrm{BR}$ \\
\hline Prof. Dr. & Marcelo Luiz Gabriel & $\mathrm{BR}$ \\
\hline Dra. & Marcilene Dianin Vianna & BR \\
\hline Dr. & Marcos Cerqueira Lima & FR \\
\hline Msc. & Maria Creuza Borges Araújo & $\mathrm{BR}$ \\
\hline Dsc. & Maria Augusta Soares Machado & BR \\
\hline Prof. LL.B. & Michael Llorénz & US \\
\hline Md. & Mihaela Stet & RO \\
\hline Msc. & Minelle Enéas Silva & $\mathrm{BR}$ \\
\hline Msc. & Moises Andrade Coelho & $\mathrm{BR}$ \\
\hline Prof. Dr. & Nilgun Tosun & TR \\
\hline Dr. & Osvaldo L. Gonçalves Quelhas & BR \\
\hline Dr. & Ozgur Dogerlioglu & $\mathrm{TR}$ \\
\hline Msc. & Paola Schmitt Figueiró & $\mathrm{BR}$ \\
\hline Dra. & Patricia Guarnieri & $\mathrm{BR}$ \\
\hline Dr. & Patrick Jaska & US \\
\hline Dr. Student & Paulo Cesar Chagas Rodrigues & $\mathrm{BR}$ \\
\hline Dr. & Paulo Afonso Lopes Da Silva & $\mathrm{BR}$ \\
\hline Dr. & Pedro Carlos Resende Jr. & $\mathrm{BR}$ \\
\hline Dr. & Pedro Paulo Andrade Júnior & $\mathrm{BR}$ \\
\hline Dna. & Raffaela Leane Zenni Tanure & $\mathrm{BR}$ \\
\hline MBA. & Rahul Pratap Singh Kaurav & IN \\
\hline MBA. & Raj Kumar Bhattarai & NP \\
\hline Ph.D. Student & Raquel Marrafon Nicolosi & ES \\
\hline Prof. Me. & Ricardo Mânica & $\mathrm{BR}$ \\
\hline Dr. & Ricardo Moreira Da Silva & $\mathrm{BR}$ \\
\hline
\end{tabular}


INDEPENDENT JOURNAL OF MANAGEMENT \& PRODUCTION (IJM\&P)

http://www.ijmp.jor.br

v. 5, n. 1, October - January 2014.

ISSN: 2236-269X

DOI: 10.14807/ijmp.v5i1.157

\begin{tabular}{lll}
\hline Dr. & Robin V. Watkins & US \\
\hline Dn. & Rodolfo Reinaldo Hermes Petter & BR \\
\hline Dra. & Sabrina Nunes Vieira & BR \\
\hline Mr. & Sandeep Bhattacharjee & IN \\
\hline Dna. & Sandra Miranda Neves & BR \\
\hline Dra. & Sandra Rufino & BR \\
\hline Ph.D. & Sanjay Sharma & IN \\
\hline Msc. & Sereen Ibrahim Abu Aisheh & PS \\
\hline Msc. & Silvia Cristiane Marangoni & BR \\
\hline Dr. Student & Sivanilza Teixeira Machado & BR \\
\hline Ms & Stella Nnedinma Ayika & NG \\
\hline Ph.D. & Tamer Mohamed Khalaf & SA \\
\hline Dra. & Tatiana Aparecida Rosa Da Silva & BR \\
\hline Ph.D. & Tatyana Valentinovna Bobra & UA \\
\hline Dr. & Tony Crespo Franco & ES \\
\hline Dr. & Trellany V. Thomas-Evans & US \\
\hline Msc. & Ursula Gomes Rosa Maruyama & BR \\
\hline Dr. & Valdecy Pereira & BR \\
\hline Prof. Dn. & Victor Freitas De Azeredo Barros & PT \\
\hline Ph.D. & Vinicius Amorim Sobreiro Sobreiro & BR \\
\hline Prof., Dr. & Vladimir Evgen'evitch Bakhrushin & UA \\
\hline Dr. & Wagner Cezar Lucato & BR \\
\hline Ph.D. Candidate & Wilda Georgine Smith & US \\
\hline Dna. & Zaida Cristiane Dos Reis & BR \\
\hline & & \\
\hline
\end{tabular}

January 1,2014

Prof. Dn. Paulo Cesar Chagas Rodrigues

Chief Editor 
SPONSORS BY 